\title{
The Surgical Nosology In Primary-care Settings (SNIPS): a simple bridging classification for the interface between primary and specialist care
}

\author{
Russell L Gruen*1, Stephanie Knox², Helena Britt ${ }^{2}$ and Ross S Bailie ${ }^{1}$
}

Address: ${ }^{1}$ Flinders University NT Clinical School \& Menzies School of Health Research, Darwin, Australia and ${ }^{2}$ AIHW General Practice Statistics and Classification Unit, Family Medicine Research Centre, University of Sydney, Sydney, Australia

Email: Russell L Gruen* - Russell.Gruen@menzies.edu.au; Stephanie Knox - sknox@med.usyd.edu.au; Helena Britt - helenab@med.usyd.edu.au; Ross S Bailie - rossb@menzies.edu.au

* Corresponding author

Published: 13 May 2004

BMC Health Services Research 2004, 4:8

This article is available from: http://www.biomedcentral.com/I472-6963/4/8

(C) 2004 Gruen et al; licensee BioMed Central Ltd. This is an Open Access article: verbatim copying and redistribution of this article are permitted in all media for any purpose, provided this notice is preserved along with the article's original URL.

\begin{abstract}
Background: The interface between primary care and specialist medical services is an important domain for health services research and policy. Of particular concern is optimising specialist services and the organisation of the specialist workforce to meet the needs and demands for specialist care, particularly those generated by referral from primary care. However, differences in the disease classification and reporting of the work of primary and specialist surgical sectors hamper such research. This paper describes the development of a bridging classification for use in the study of potential surgical problems in primary care settings, and for classifying referrals to surgical specialties.
\end{abstract}

Methods: A three stage process was undertaken, which involved: (I) defining the categories of surgical disorders from a specialist perspective that were relevant to the specialist-primary care interface; (2) classifying the 'terms' in the International Classification of Primary Care Version 2Plus (ICPC-2 Plus) to the surgical categories; and (3) using referral data from 303,000 patient encounters in the BEACH study of general practice activity in Australia to define a core set of surgical conditions. Inclusion of terms was based on the probability of specialist referral of patients with such problems, and specialists' perception that they constitute part of normal surgical practice.

Results: A four-level hierarchy was developed, containing 8, 27 and 79 categories in the first, second and third levels, respectively. These categories classified 2050 ICPC-2 Plus terms that constituted the fourth level, and which covered the spectrum of problems that were managed in primary care and referred to surgical specialists.

Conclusion: Our method of classifying terms from a primary care classification system to categories delineated by specialists should be applicable to research addressing the interface between primary and specialist care. By describing the process and putting the bridging classification system in the public domain, we invite comment and application in other settings where similar problems might be faced. 


\section{Background}

The interface between primary care and specialist medical services is increasingly the focus of health care quality and equity concerns. Understanding referrals and optimising referral pathways, deciding which conditions are most efficiently managed in primary care and which are better managed by specialists, and working out how specialists can best support primary care practitioners are some of the aims of modern health system improvement initiatives [1].

An example is the use of metropolitan-based specialist surgical services to meet the needs of rural, remote and disadvantaged Indigenous populations in Australia. A current concern is whether or not visiting specialists improve access and health outcomes, and change the nature of referrals from primary care practitioners, when compared with hospital-based services alone. To answer these questions, we designed a population-based study of the management of patients with surgical problems who presented to primary care.

The planned study, however, faced difficulties classifying surgical disorders. The problems arose because the data were collected in primary care, but the information was to be used for planning specialist services. We sought a means of identifying potential specialist surgical conditions managed in primary care, and then classifying them into categories that described the potential needs and demand for surgical specialists, acknowledging the way that the specialist workforce is organised. No existing classification system had these attributes.

Throughout the world, the surgical workforce is primarily organised in anatomical disciplines (e.g. colorectal surgery, cardiothoracic surgery, head and neck surgery). Even disciplines based on a 'physiological system', such as orthopaedic surgery, tend to be subdivided into anatomically-based sub-specialties (e.g. hand, spine, hip and knee). Surgical work is classified accordingly, with an emphasis also on procedures (e.g. colectomy, angioplasty, fine needle aspiration of the thyroid). Classification systems of surgical disorders, used for individual and hospital audit, reflect the specialists' anatomical and procedural orientations [2].

In contrast, primary care practitioners have emphasised the holistic nature of their work, and have resisted reductionist anatomical or pathological approaches to classification [3]. The International Classification of Primary Care (ICPC) [4] and the Read Clinical Codes [5] are examples. ICPC has a biaxial structure, based primarily on physiological body systems, and designed specifically for classifying the reasons for encounters, diagnoses or problems, and the processes of care. In primary care ICPC has considerable benefits over the International Classification of Diseases (ICD) for classifying problems that do not (yet) have a precise diagnosis, as well as administrative tasks and care processes such as referrals, tests and procedures [6]. The emphasis of ICD on disease, even in its $10^{\text {th }}$ edition, means that a high proportion of primary care visits end up classified in residual categories $[7,8]$.

Neither ICPC, with its emphasis on physiological body systems, nor ICD, with its emphasis on diseases, organise potential surgical problems along the anatomical lines on which the structure of surgical services is based. Existing 'maps' between ICPC and ICD do not overcome this problem. As a result, practitioners, policy-makers and researchers who are interested in the interface between primary and specialist surgical care have no standard way of classifying disorders.

What was required for our research, and which did not exist, was a straight-forward classification in a simple hierarchy organised according to surgical disciplines, but which facilitated effective coding of conditions encountered in primary care environments. Hence we developed the Surgical Nosology In Primary-care Settings (SNIPS). We have used the classification system in analysis of a national dataset of general practice activity to describe referrals to surgeons [9] and to study the influence of specialist proximity on referral rates [10]. In this article we describe the classification and its development, with the aim of illustrating the process by which terms developed for primary care were classified in a new way to allow study of the role of specialists.

\section{Methods}

The development of SNIPS had three stages: (1) defining categories of disorders from a specialist surgical perspective; (2) choosing a primary care terminology and classifying it using the surgical categories; and (3) employing a large national dataset of general practice activity to improve the precision of the classification by restricting the nosology to disorders that are consistently referred to surgeons.

\section{Defining categories of surgical disorders from a specialist perspective}

A panel of specialists at a large general hospital, Royal Darwin Hospital (RDH), in northern Australia, was convened to reach agreement on the major categories of surgical disorders. The panel included two general surgeons, an orthopaedic surgeon, an ophthalmologist, an obstetrician-gynaecologist, and an ENT registrar, all of whom were actively involved in both inpatient and outpatient care. Other specialists' advice was sought when necessary. The panel was asked to delineate the main categories of conditions and work in each of their specialties. For 
guidance, they were asked to consider the way conditions and procedures are grouped in subspecialty societies, and when presented at conferences or in journals and textbooks.

After an initial draft, the panel agreed that a four-level single axial hierarchy would be economical and still provide specificity for the most important groups of surgical disorders. The top three levels were to be delineated by the panel, and existing terms designed for primary care were to be used as the fourth level. The names of the major surgical specialties (e.g. general surgery, orthopaedic surgery) were allocated to Level 1 . The first, second and third levels were given three, four and five-letter codes respectively, while the fourth level retained the original six-digit codes of the primary care terminology. An example of related Level 1, 2 and 3 categories is "Orthopaedics" (ORT), "Shoulder" (SHLD) and "Shoulder Injury/Instability" (SHINJ), to which terms for "Sprain;shoulder" (L79021), "Injury;rotator cuff" (L79041) and "Dislocation;shoulder" (L80003), among others, were classified. Another Level 3 category under "Shoulder" is "Shoulder Arthritis/ Pain" (SHART), to which terms concerning osteoarthritis, rheumatoid arthritis, rotator cuff syndrome and frozen shoulder were classified.

We aimed for the hierarchy to meet the criteria for a robust classification, especially that the structure is appropriate for the majority of referrals from primary care; that it makes sense to specialists in all surgical disciplines; that categories are mutually exclusive; that each term has a place and that there is only one place for each term [11].

In development of the hierarchy some disciplinary areas were discrete and some overlapped. Where the overlap was such that several of the subcategories may be routinely managed by one or other of these specialties, the specialties were combined as one heading. Specialties whose work derives mostly from sources other than primary care, such as cardiothoracic surgery and transplant surgery, were grouped as "Other specialities". To maintain similar specificity at each level in each specialty, the panel decided that a three-level classification was not required in ENT surgery, urology, ophthalmology and obstetrics, in which cases Level 2 was omitted. Finally, a few Level 2 and Level 3 categories could have been allocated to more than one parent category. Examples included the allocation of hand surgery to 'Forearm, wrist and hand' under orthopaedics rather than plastic or general surgery, and thyroid surgery to breast/endocrine not ENT surgery. In such cases consensus about the most appropriate allocation was reached through panel discussion.

\section{Classification to the surgical categories of terms from an existing primary care nosology}

We used terms from the International Classification of Primary Care, Version 2-Plus (ICPC-2 Plus) $[12,13]$ for three main reasons. First, the validity and reliability of ICPC as a research tool in primary care have been established [13-15]. Second, ICPC-2 Plus contained, at the time of this work, an extended vocabulary of 7640 'terms' derived from terminology used in over 500,000 general practice encounters. These terms greatly enhance the specificity of coding compared to what was possible with the 1388 higher-order 'rubrics' in ICPC-2. Third, ICPC-2 Plus has been used for the study of general practice activity in Australia since 1998 [16-18] and a database was available to facilitate development of our nosology.

Furthermore, as part of ICPC-2 Plus, the Family Medicine Research Centre at the University of Sydney has developed an electronic interface providing easy keyword-prompted selection of appropriate ICPC-2 Plus terms and facilitating its integration into other databases [see: http:// www.fmrc.org.au]. In addition, ICPC has widespread international use, and has been implemented in resourcepoor settings where outreach, the subject of our research, is likely to be particularly relevant.

The codes for ICPC-2 Plus terms consist of a letter and five digits. For example, cataract can be coded as 'Cataract' (F92001), 'Cataract;senile' (F92002) and 'Lenticular opacities' (F92003). The letter indicates the ICPC-2 'chapter', organised mostly in body systems ('F' refers to the Eye). The letter plus two digits comprise the ICPC-2 'rubric', or major concept being represented (in this case, 'cataract'). The remaining three digits delineate terms that enable variations in and different representations of these rubrics to be coded more accurately. Due to their greater specificity, we classified the ICPC-2 Plus terms (rather than the broader ICPC- 2 rubrics) to the Level 3 categories.

Reference was made to the Bettering Evaluation And Care of Health (BEACH) study, the largest analysis of general practice activity in Australia, and which used ICPC-2 Plus for coding problems and activities. In the ongoing $\mathrm{BEACH}$ study approximately 1000 GPs randomly selected from all practising GPs in Australia are enrolled each year, and each enrolled GP prospectively records information regarding 100 consecutive encounters with patients, including the problems managed and new referrals made [17]. The BEACH data is cross-sectional and encounterbased.

In the development of SNIPS, we used the first three years of the BEACH data (1998-2001), which included 303,000 patient encounters and the 'terms' describing 449,277 problems managed. We allocated each term that 
had been recorded at least once in the dataset to the most appropriate Level 3 category. Conditions that could only be related indirectly to surgical specialties were coded to the group most likely to be relevant (e.g. 'Diabetes noninsulin dependent' to 'diabetes/systemic/retinal' under ophthalmology). Any that were obviously unrelated to surgery (eg Psoriasis) were categorised as 'Non-surgical', and those that were too non-specific to determine (eg 'Pain:arm') were categorised as 'Other.'

\section{Restriction to a 'core set' of surgical problems using national referral data}

At this stage a preliminary mapping of any potential surgical terms had been achieved. The final stage of development of the classification aimed to define a restricted set of 'surgical problems' by examining which problems had been referred to surgeons in the BEACH study. Referrals were counted if they were coded in ICPC-2 Plus as having been made to any of a broad range of specialties in which surgical procedures form a large part of practice, including endoscopy and clinics usually attended by surgeons or obstetricians, such as breast, antenatal and in-vitro fertilisation clinics. In principle, surgical problems could be defined as those problems that a large, nationally-representative sample of GPs refers to surgical specialists via private clinics, hospital outpatients and emergency departments.

In practice, while this generally held true, there was also a considerable amount of 'noise' in the data, particularly from the very common presentations to general practice, such as hypertension and depression, which were recorded as being referred to a surgical specialist in a very small number of cases. Explanations for these aberrations undoubtedly lie in the fact that problems in primary care are not always discrete. Consider an elderly patient with multiple problems who has had trouble with her vision and who is depressed. Her poor vision may be contributing to her depression, and as part of its treatment, a referral to an ophthalmologist may be arranged. It is easy to see how, in this case, the problem of 'depression' can result in a specialist ophthalmological referral. However the rate of these referrals, relative to the rate of management of depression in primary care, for example, is trivial. The implication of having depression in the classification is that while it is not a surgical condition in any real sense, it could lead to many more encounters being included in studies of surgical problems. Analyses would therefore use larger denominators, and have lower estimates of referral rates and potentially less precise estimates. It was clear that inclusion of problems such as depression would reduce the usefulness of the nosology.

To improve the precision for 'real' surgical problems we defined a threshold percentage of total encounters with a problem in which a new surgical referral was initiated. Terms for which the percentage referred was above the threshold were automatically included. Terms not referred at all were excluded. Terms for which the percentage referred was between zero and the threshold value were then submitted to the most appropriate member of the panel and included only if he or she indicated that patients with that particular problem would be "a usual part of practice in that specialty." Prior to defining the terms to send to the panel we performed a sensitivity analysis using threshold percentages of $0,0.01 \%, 1 \%, 5 \%$, $10 \%, 15 \%$ and $20 \%$. A threshold percentage was chosen to maximise both the number of surgical referrals in the data and the percentage of terms in the data that were referred.

\section{Results}

Table 1 shows the three-level hierarchy agreed upon by the specialist panel. Orthopaedic surgery, urology, ENT, ophthalmology and obstetrics and gynaecology were defined as relatively discrete top-level groupers. On the other hand, general surgery, vascular surgery and plastic surgery were grouped together as one top-level category, because it was evident that several of the subcategories may be routinely managed by one or other of these specialties. Specialties such as neurosurgery, cardiothoracic surgery, and transplant surgery, whose work derives mostly from sources other than primary care, were grouped under "Other specialities". Examples of grouping decisions that were debated included the allocation of hand surgery to 'Forearm, wrist and hand' under orthopaedics rather than plastic or general surgery, and thyroid surgery to "Breast/endocrine" under general surgery, not ENT surgery.

Figure 1 shows the sensitivity analysis for setting a threshold percentage for inclusion of a term in the core set of surgical conditions. A threshold of ten percent was chosen as maximising both the number of referrals in the BEACH data and the percentage of all patients in BEACH who were referred.

After the panel allocated the terms referred at between zero and $10 \%$ of the presentations, when compared with the automatic $10 \%$ threshold, the total number of terms increased from 1,119 to $2,050(+83 \%)$, the total number of referrals increased from 10,481 to $13,570(+29 \%)$, the total number of problems managed by GPs increased from 44,830 to $143,013(+219 \%)$, and the percentage of problems referred fell from $23 \%$ to $9.5 \%$ (a reduction of $59 \%$ ). We were prepared to accept compromises in specificity for the benefits of improved sensitivity. 


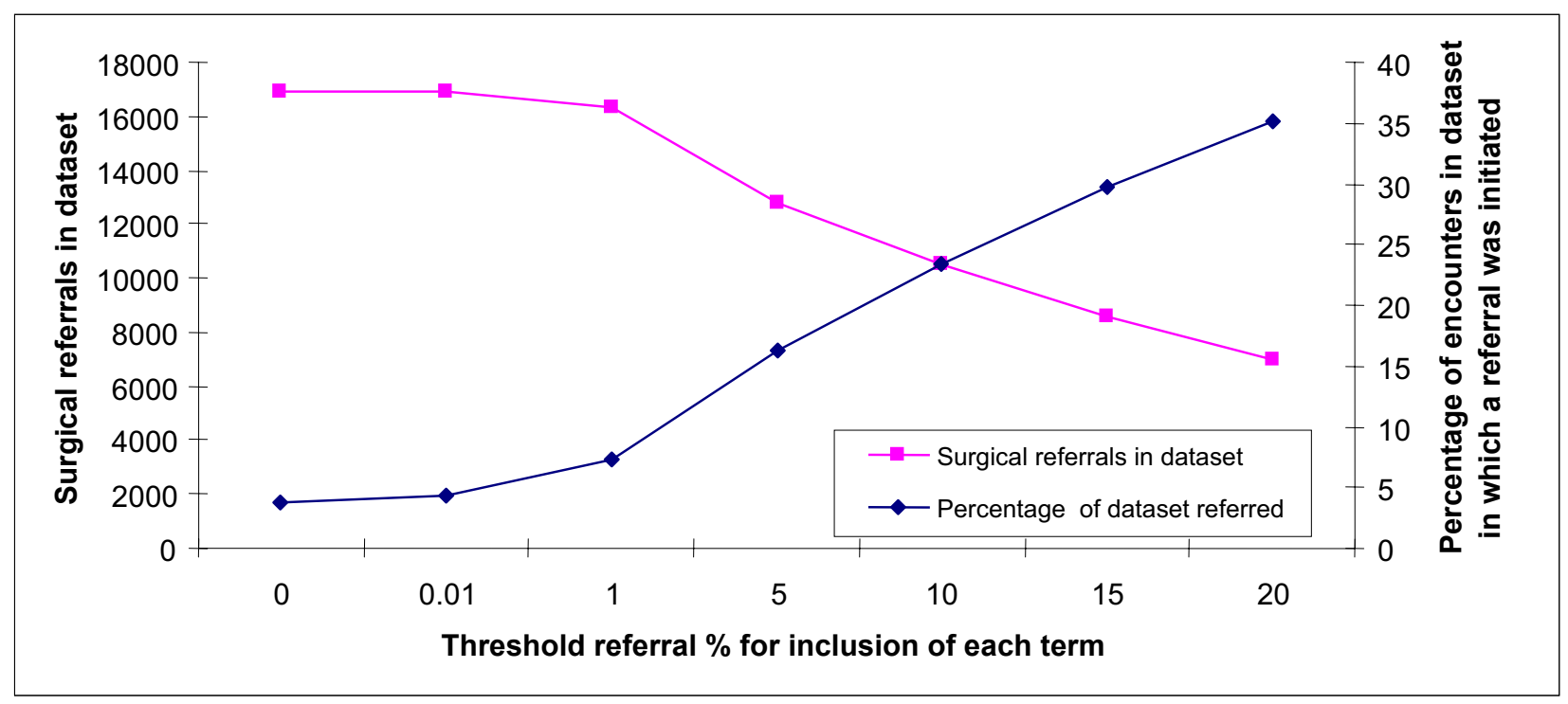

Figure I

Sensitivity analysis of setting a threshold referred percentage for inclusion in the core set of surgical problems.

Table I: Structure of SNIPS and the number of ICPC-2 Plus terms it classifies

\begin{tabular}{|c|c|c|c|c|c|}
\hline Level I & Level 2 & L2 Code & Level 3 & L3 Code & Terms \\
\hline \multirow[t]{20}{*}{ General/Vascular/Plastics (GVP) } & Hepatobiliary-Upper GI & HBUG & Upper GI & UPRGI & 81 \\
\hline & & & Hepatobiliary-Pancreatic & HPBPA & 31 \\
\hline & & & Small bowel/Appendix & SBAPP & 16 \\
\hline & Colorectal & CREC & Colon Neoplasia/Bleeding & CLNCA & 15 \\
\hline & & & Colonic Other & COLON & 49 \\
\hline & & & Perianal & PANAL & 22 \\
\hline & Hernia & HERN & Inguinal hernia & HEING & 4 \\
\hline & & & Ventral hernia & HERVN & 7 \\
\hline & Abdominal other & ABDO & Generalised Abdominal Pain & ABDPN & 18 \\
\hline & & & Anaemia \& Lymphoid & ANLYM & 18 \\
\hline & & & Abdominal other 2 & ABDOM & 42 \\
\hline & Breast-Endocrine & BREN & Breast Lump/Cancer & BRLMP & 20 \\
\hline & & & Breast Other & BREST & 42 \\
\hline & & & Endocrine & ENDOC & 34 \\
\hline & & & Obesity & OBESI & 5 \\
\hline & Skin/Integument & SKIN & Skin Infection/Injury & INFNJ & 91 \\
\hline & & & Skin Lesions & LSION & 75 \\
\hline & Vascular & VASC & Arterial & ARTRL & 29 \\
\hline & & & Varicose Veins & VEINS & 9 \\
\hline & & & Venous thrombosis & DEVET & 8 \\
\hline
\end{tabular}


Table I: Structure of SNIPS and the number of ICPC-2 Plus terms it classifies (Continued)

\begin{tabular}{|c|c|c|c|c|c|}
\hline \multirow[t]{16}{*}{ Orthopaedics (ORT) } & Back & BACK & Back & SPINE & 122 \\
\hline & Shoulder & SHLD & Shoulder Arthritis/Pain & SHART & 20 \\
\hline & & & Shoulder Injury/Instability & SHINJ & 7 \\
\hline & Forearm, Wrist \& Hand & FWRH & Forearm fracture & FAFRC & II \\
\hline & & & Forearm/Hand Other Injury & FAINJ & 15 \\
\hline & & & Forearm/Hand Non-Injury & FAOTH & 30 \\
\hline & Hip Arthritis & HIPA & Hip Arthritis & HPART & 8 \\
\hline & Knee & KNEE & Knee Arthritis/pain & KNART & II \\
\hline & & & Knee Injury/instability & KNINJ & 27 \\
\hline & Ankle \& Foot & ANKF & Ankle Fracture & ANFRC & 7 \\
\hline & & & Ankle/Foot Other Injury & ANINJ & 13 \\
\hline & & & Ankle/Foot Non-Injury & ANOTH & 26 \\
\hline & Ortho Other & ORTO & Other Arthritis (Site NOS) & OTART & 22 \\
\hline & & & Other Fracture/Dislocation & OTFRC & 31 \\
\hline & & & Other Injury (Non-Frac/Disl) & OTINJ & 28 \\
\hline & & & Other Ortho Non-Injury & ОТОТ̈н & 92 \\
\hline \multirow[t]{6}{*}{ Ear, Nose \& Throat (ENT) } & - & ENT2 & Ear/Mastoid Infective & EARIN & 34 \\
\hline & & & Ear/Mastoid Non-Infective & EAROT & 69 \\
\hline & & & Nose/Paranasal Sinuses & NOSSI & 26 \\
\hline & & & Tonsils/Adenoids & TONSL & 13 \\
\hline & & & Larynx/pharynx & LARYP & 19 \\
\hline & & & Oro-facio-maxillary & FACMX & 36 \\
\hline \multirow[t]{10}{*}{ Urology (URL) } & - & UROL & Stones/upper tract obstruction & STOUP & 18 \\
\hline & & & Haematuria/Urothelial/renal tumours & HMTCC & 17 \\
\hline & & & Bladder dysfunction & BLDYS & 21 \\
\hline & & & Bladder outflow obstruction & BOOBS & 24 \\
\hline & & & Prostate cancer & PRSCA & 5 \\
\hline & & & Impotence \& Penile & IMPEN & 25 \\
\hline & & & Testicle, Scrotum \& Vas & TSTIC & 33 \\
\hline & & & Paediatric urology & PAEDU & 14 \\
\hline & & & Urinary Tract Infection & URUTI & 6 \\
\hline & & & Urology Other & UROTH & 42 \\
\hline \multirow[t]{10}{*}{ Eye (EYE) } & - & EYE2 & Painful/red eye & PNRED & 17 \\
\hline & & & Visual Loss & VSLOS & 29 \\
\hline & & & Cataract & CTRCT & 4 \\
\hline & & & Glaucoma & GLAUC & 4 \\
\hline & & & Diabetes, systemic \& retinal & DBRET & 10 \\
\hline & & & Muscle imbalance \& squint & SQINT & 11 \\
\hline & & & Eye Trauma & EYINJ & 16 \\
\hline & & & Lid/conjunctival disorders & LDCON & 31 \\
\hline & & & Refraction & REFRC & 15 \\
\hline & & & Eye Other & EYOTH & 33 \\
\hline \multirow[t]{5}{*}{ Gynaecology (GYN) } & Cancer/ Precancer & CAPR & Cervical Cancer/Precancer & CERCA & 24 \\
\hline & & & Other Gynae Neoplasia & GYNCA & 12 \\
\hline & Menstrual abn/ Pelvic pain & MENS & Menstrual Disorders & MENST & 27 \\
\hline & & & Pelvic Pain & PELPN & 22 \\
\hline & General Gynaecology & GGYN & Prolapse & GYPRL & 8 \\
\hline
\end{tabular}


Table I: Structure of SNIPS and the number of ICPC-2 Plus terms it classifies (Continued)

\begin{tabular}{|c|c|c|c|c|c|}
\hline & & & Other General Gynae & GYOTH & 59 \\
\hline & \multirow[t]{2}{*}{ Fertility } & \multirow[t]{2}{*}{ FERT } & Infertility F & INFRT & 6 \\
\hline & & & Fertility Control F & STRLN & 11 \\
\hline \multirow[t]{4}{*}{ Obstetrics (PRG) } & \multirow[t]{4}{*}{-} & \multirow[t]{4}{*}{ PREG } & Antenatal Normal & ANTEN & 18 \\
\hline & & & Antenatal Abnormal & ANTAB & 49 \\
\hline & & & Delivery & LABOR & 16 \\
\hline & & & Postnatal & PSTNT & 13 \\
\hline \multirow[t]{5}{*}{ Other Specialties (OTH) } & \multirow[t]{2}{*}{ Neurosurgery } & \multirow[t]{2}{*}{ NEUR } & Head Injury & HDINJ & 18 \\
\hline & & & Neurosurgery Other & NEOTH & 41 \\
\hline & Thoracic surgery & THOR & Thoracic & THOTH & 28 \\
\hline & Other Injury & INJU & Injury Multiple \& NOS & INJOT & 11 \\
\hline & Other Non-Injury & OTHE & Other Non-injury & OTHER & 49 \\
\hline
\end{tabular}

Table 2: Problems and referrals in the final classification of surgical problems

\begin{tabular}{lcccc}
\hline & Level I & Level 2 & Level 3 & Terms \\
\hline $\begin{array}{l}\text { No. of categories } \\
\text { Total no. patient encounters with GPs per category } \\
\quad \text { Median }\end{array}$ & 8 & 27 & 79 & 2050 \\
$\quad$ Range & 6414 & 3647 & 838 & 11 \\
$\begin{array}{l}\text { Total no. referrals per category } \\
\quad \text { Median }\end{array}$ & $4060-58275$ & $378-26958$ & $74-15016$ & $1-4394$ \\
$\quad$ Range & 1284 & 391 & 142 & $14-923$ \\
\hline
\end{tabular}

( $n=143,013$ in restricted surgical dataset)

Table 2 summarises the number of categories, and the median and range of the number of problems and referrals from the $\mathrm{BEACH}$ database that were classified in each category and used in the development of SNIPS. The complete classification is included in the accompanying 1 (SNIPS.MDB).

\section{Discussion}

We have developed a new classification of an existing primary care terminology to facilitate integration and coordination between primary care and specialist sectors that have traditionally had language and classification differences. SNIPS should facilitate study of the potential and actual need and demand for surgical services generated by problems seen in and referred from primary care. Understanding the potential and actual scope and volume of surgical work is important for policy, planning and research purposes. Although we were specifically interested in surgical specialties, the empirical approach used in developing this classification may also apply to nonsurgical specialties.

\section{Applications}

SNIPS has been used in three ways to date. The first was to interrogate the BEACH data, already coded in ICPC-2 Plus terms, to describe in detail referrals to surgical specialists in a national sample [9]. The aim was to inform policy regarding surgical workforce distribution and training requirements, and the data were presented in the SNIPS Level 1, 2 and 3 categories. The second was to identify potential surgical problems in the same dataset and then determine whether proximity of a specialist influenced general practitioners' referral rates [10]. SNIPS was used to define and limit referrals that were to be included in the analysis, and to facilitate analysis of individual specialties separately. The third way SNIPS has been used, and the original impetus for its development, was to classify data collected in a primary care setting for a population-based 
study of the impact of specialist outreach visits on referrals and access to specialist care. A simple 'look-up' engine involving the ICPC-2 Plus keywords was developed, data were stored as ICPC-2 Plus terms in a Microsoft Access database, and results were reported for Levels 1, 2 and 3 as required.

\section{Limitations of SNIPS}

Issues of structure

SNIPS was developed through a combination of consensus and data-driven pragmatism, but the structure reflected the opinions of a small number of specialists. For example, sub-classification of specialties such as cardiothoracic surgery, which receives relatively few referrals directly from general practitioners, suited the intended application to primary care settings. It is possible, however, that other surgeons will have different ways of categorising surgical disorders. We believe our system is robust for our requirements, but acknowledge that it may not be the only way.

\section{Issues of inclusion}

Essentially our classification defines surgical disorders using both specialists' and primary carers' input - specialists by defining the structure, primary carers by their description, and the subsequent classification of that description, of who they referred. It was intended that the classification be broadly inclusive. As such, we sacrificed some specificity for the sake of sensitivity. Some terms may seem, at best, to be indirectly related to surgery. The process of setting a threshold for the percentage referred, and then selecting on their merits terms which had a lower percentage, was an attempt to improve on an automated process governed by the data.

\section{Issues of exclusion}

ICPC-2 was originally developed with the intention that 'rubrics' would represent single concepts, but we chose to use ICPC-2 Plus terms because they offered greater specificity. On account of both the data and panel deliberations, there were many rubrics which contained both included and excluded terms. We were only concerned with terms, so this caused no problems. Any analysis at the level of ICPC-2 rubrics, however, would need to take this into account.

\section{Issues of unclassifiability}

Because ICPC-2 Plus is designed for primary care, there were many terms that could have been classified as a surgical problem in a particular situation if presenting in a particular way. While we aimed to be broadly inclusive, terms such as 'fever' for which fewer than $10 \%$ of encounters resulted in a referral and which the panel considered not a routine part of their practice, were excluded as being unclassifiable without further information.

\section{Issues of omission}

The top three levels of SNIPS cover most of the spectrum of surgical disorders but, given that ICPC-2 Plus is not a comprehensive surgical coding system, surgical conditions are represented with less precision than they may be in ICD or a surgical-procedure-based classification. While it was not apparent that this has led to omission of any conditions, it should be borne in mind that SNIPS is designed for classification of surgical presentations in primary care settings and is not a comprehensive surgical classification in its own right. Therefore SNIPS is a tool for use in studies of need and demand for surgical disorders in primary care, and is not a replacement for surgical audit classifications that are designed specifically for describing surgical procedures and treatment [2].

Furthermore, SNIPS does not capture secondary referrals from other specialists to surgeons. Cardiac valve dysfunction is an example in which an initial general practitioner's referral may be made to a medical specialist who subsequently refers the patient to a cardiac surgeon. The majority of Australia's surgical demand, however, comes from primary elective or emergency referrals from general practitioners.

Because the BEACH data is cross-sectional and encounterbased, not longitudinal and patient-based, any particular patient may have had a new referral made at a previous or subsequent encounter that is not identified in the dataset. The percentage of encounters for each term that resulted in referral may therefore underestimate the actual referral rate of problems. However, given the size of the dataset and our inclusive approach to classifying ICPC-2 Plus terms, we believe no important terms have been omitted.

\section{Issues of incorrect classification}

Every care has been taken to avoid misclassification of ICPC-2 Plus to the upper levels. Some specialists, however, may disagree with the upper hierarchy and the allocation of certain sub-specialties (as in the 'hand' and 'thyroid' examples given earlier).

One of the benefits of using ICPC-2 Plus - the ability to classify many problems which did not yet have a precise diagnosis - may also contribute to some misclassification. For example, undifferentiated abdominal pain may reflect a general surgical problem, a urological problem, a gynaecologic problem, and so on. Our specialist panel debated and reached agreement on allocation of 'symptomatic terms,' however such allocation necessarily involves greater potential for error than the classification of established diagnoses. It will be useful to determine, through further use and ongoing critique of SNIPS, whether these issues are important to its application. 


\section{Issues of generalisability}

The hierarchical structure of SNIPS is intended to be relevant to a broad range of settings. For example, in areas where only general surgical services are available, the classification of ICPC-2 Plus terms to Level 1 categories may be of greatest interest. On the other hand, where several surgical sub-specialties are available, the classification of ICPC-2 Plus terms to Levels 2 or 3 categories may be most relevant.

By taking an empirical approach using a database of referrals, SNIPS was subject to the referral practices of the 3030 participating general practitioners. The decision to refer a patient can be complex, and considerable variability in referral rates has been demonstrated between general practitioners [19-22]. However the data on which the SNIPS classification was based included referrals from a wide spectrum of solo and group practices, private clinics and community health centres, and from urban and rural parts of Australia. Given that the BEACH dataset contained 449,277 problems managed, and that every ICPC2 Plus term describing a problem that was referred to a surgeon at least once was either automatically included or submitted for panel evaluation, we erred on the side of being overly inclusive when defining 'surgical problems.' We anticipate that this will enhance the generalisability of SNIPS in settings with differing casemix, primary care provider and practice characteristics, and where the surgical workforce may be either organised differently or have different roles.

\section{Conclusions}

SNIPS was developed through an iterative process, involving several specialists and analysis of a large national dataset of general practice activity. It facilitates the practical application of data, collected using an existing primary care terminology, to issues of need and demand for specialist surgical services. We have already applied it in studies examining the primary care-specialist interface in Australia. We wish to make the system available for use in other settings for research and planning purposes, and invite discussion about the process and the outcome of its development.

\section{Competing interests}

None declared.

\section{Authors' contributions}

RG coordinated development of the classification system and wrote the main draft. SK conducted analyses. HB coordinates the BEACH program and provided expertise regarding classification. $\mathrm{RB}$ critiqued several drafts and contributed to the conceptual development of the classification and the paper.

\section{Additional material}

\section{Additional File 1}

The SNIPS classification is presented as two tables in the attached Microsoft Access 2002 database file. The table "Level123Groupers" delineates the upper three levels of the classification, with fields in the order: $<$ Level1Code $><$ Level1Name $><$ Level2Code $><$ Level2Name $>$ $<$ Level3Code $><$ Level3Name $>$ The table "SNIPS" presents the 2050 ICPC-2 Plus terms mapped to the upper three levels, with fields in the order: $<$ TermCode $><$ Term Name $><$ Level1Code $><$ Level2Code $>$ $<$ Level3Code $><$ Injury $><$ Rubric $>$

Click here for file

[http://www.biomedcentral.com/content/supplementary/14726963-4-8-S1.mdb]

\section{Acknowledgements}

The authors wish to thank the specialists and registrars at Royal Darwin Hospital for their contribution to development of the classification, and the 3030 GPs who participated in the first 3 years of the BEACH program, which is conducted by the General Practice and Statistics Unit, a collaborating unit of the Australian Institute of Health and Welfare and the University of Sydney. Over the 1998-200I period the program has been funded by the Commonwealth Department of Health and Aged Care, the Commonwealth Department of Veterans' Affairs (1998-99), the National Occupational Health and Safety Commission (1998-99), Astra Zeneca (Aust.), Aventis-Pharma Pty Ltd, Roche Products Pty Ltd, and Janssen-Cilag Pty Ltd (200I).

Dr Gruen is supported by a National Health and Medical Research Council Medical Postgraduate Scholarship, and a Surgeon Scientist Award from the Royal Australasian College of Surgeons Foundation.

\section{References}

I. Katon W, Von Korff M, Lin E, Simon G: Rethinking practitioner roles in chronic illness: the specialist, primary care physician, and the practice nurse. Gen Hosp Psychiatry 200I, 23:138-I 44.

2. Clinical audit research unit: The Otago Clinical Audit System 2003 [http:/ /www.otago.ac.nz/ouaudit/]. Dunedin: University of Otago

3. WONCA International Classification Committee: The International Classification of Primary Care 2nd edition. Oxford: Oxford University Press; 1998.

4. Lamberts H, Wood M: International Classification of Primary Care (ICPC) Oxford: Oxford University Press; 1987.

5. NHS Information Authority: The Clinical Terms Version 3 (The Read Codes) Birmingham: NHS Information Authority; 1998.

6. World Health Organization: The International Statistical Classification of Diseases and Related Health Problems, tenth revision (ICD-I0) Geneva: World Health Organization; 1992.

7. Spasoff RA: Epidemiologic methods for health policy New York: Oxford University Press; 1999:72.

8. Starfield B: Primary care: balancing health needs, services, and technology New York: Oxford University Press; 1998:355.

9. Gruen RL, Knox S, Carson P, O'Rourke IC, Britt H, Bailie RS: The demand for surgery: an analysis of referrals from Australian general practitioners. Aust NZJ Surg in press.

10. Gruen RL, Knox S, Britt $H$, Bailie RS: Where there is no surgeon: the effect of specialist proximity on general practitioners' referral rates. Med J Aust 2002, 177: III-II5.

II. Cimino JJ, Clayton PD, Hripcsak G, Johnson SB: Knowledge-based approaches to the maintenance of a large controlled medical terminology. J Am Med Inform Assoc 1994, 1:35-50.

12. Classification Committee of the World Organisation of Family Doctors (WONCA): ICPC-2: International Classification of Primary Care 2nd edition. Oxford: Oxford University Press; 1998. 
13. Britt $\mathrm{H}$, Angelis $\mathrm{M}$, Harris $\mathrm{E}$ : The reliability and validity of doctor-recorded morbidity data in active data collection systems. Scand J Prim Health Care 1998, 16:50-55.

14. Britt $\mathrm{H}$ : A new coding system for computerised clinical systems in primary care - ICPC plus. Aust Fam Physician 1997, 26(Suppl 2):S79-S82.

15. Britt $\mathrm{H}$ : Reliability of central coding of patient reasons for encounter in general practice, using the International Classification of Primary Care. Journal of Informatics in Primary Care 1998:3-7.

16. Britt H, Sayer GP, Miller GC, Charles J, Scahill S, Horn F, Bhasale A, McGeechan K: BEACH - Bettering the evaluation and care of health. $A$ study of general practice activity, six-month interim report (No. GEP I) Canberra: Australian Institute of Healthf and Welfare; 1999.

17. Britt H, Miller GC, Charles J, Knox S, Sayer GP, Valenti L, Henderson J, Kelly Z: General practice activity in Australia 1999-2000 (No. GEP 5) Canberra: Australian Institute of Health and Welfare; 2000.

18. Britt H, Miller GC, Valenti L: 'It's different in the bush' - a comparison of general practice activity in metropolitan and rural areas of Australia 19982000 (No. GEP 6) Canberra: Australian Institute of Health and Welfare; $200 \mathrm{I}$

19. Newton J, Hayes $V$, Hutchinson $A$ : Factors influencing general practitioners' referral decisions. Fam Pract 1991, 8:308-313.

20. Noone A, Goldacre M, Coulter A, Seagroatt V: Do referral rates vary widely between practices and does supply of services affect demand? A study in Milton Keynes and the Oxford region. J $R$ Coll Gen Pract 1989, 39:404-407.

21. Roland MO, Bartholomew J, Morrell DC, McDermott A, Paul E: Understanding hospital referral rates: a user's guide. $B M$ 1990, 301:98-102.

22. Fertig A, Roland $\mathrm{M}$, King $\mathrm{H}$, Moore $\mathrm{T}$ : Understanding variation in rates of referral among general practitioners: are inappropriate referrals important and would guidelines help to reduce rates? $B M]$ 1993, 307:| 1467-I470.

\section{Pre-publication history}

The pre-publication history for this paper can be accessed here:

http://www.biomedcentral.com/1472-6963/4/8/prepub
Publish with Bio Med Central and every scientist can read your work free of charge

"BioMed Central will be the most significant development for disseminating the results of biomedical research in our lifetime. "

Sir Paul Nurse, Cancer Research UK

Your research papers will be:

- available free of charge to the entire biomedical community

- peer reviewed and published immediately upon acceptance

- cited in PubMed and archived on PubMed Central

- yours - you keep the copyright 